\title{
The Hidden Colors of Coronavirus: the Burden of Attributable COVID-19 Deaths
}

J Gen Intern Med 36(5):1463-5

DOI: $10.1007 / \mathrm{s} 11606-020-06497-4$

(C) Society of General Internal Medicine 2021

\section{INTRODUCTION}

The USA leads the world in the number of COVID-19 cases and deaths. ${ }^{1}$ However, COVID-19 has not impacted all communities equally. Prior reports have shown higher rates of COVID-19 cases and deaths in Black, Latinx, and American Indian/Alaska Native (AIAN) communities; ${ }^{2}$ data for Asians and Native Hawaiian Pacific Islander (NHPI) are aggregated or non-existent, leaving disparities within these communities of color unknown and, thus, invisible. Using a dataset that disaggregates and reports Asian and NHPI deaths by age, we evaluate the attributable COVID-19 mortality burden on Asians, NHPIs, and other racial/ethnic groups of color, in comparison to non-Hispanic Whites.

\section{METHODS}

We used data from the National Center for Health Statistics (NCHS), ${ }^{3}$ which provides weekly updated, national data on the count and distribution of COVID-19 deaths and total deaths by age and race/ethnicity. Data included COVID-19 deaths between February 1 and October 10, 2020.

We examined the burden of disease from COVID-19 using attributable mortality, which accounts for not only the effect of infection on mortality but also the prevalence within the population of interest. ${ }^{4}$ We defined COVID-19 attributable death as the proportion of COVID-19 deaths out of the total number of deaths from all causes, including COVID-19, for each group of focus. We compared the attributable death from COVID-19 by known race/ethnicity overall, and then across age categories. Hispanic is a mutually exclusive group from race; all racial groups are tabulated as non-Hispanic. To determine whether differences by race/ethnicity were statistically significant, we regressed attributable mortality on race as a categorical variable with non-Hispanic Whites as the referent.

This manuscript has not been previously published elsewhere and is not under consideration in the same or substantially similar form in any other peer-reviewed media.

Received August 9, 2020

Accepted December 17, 2020

Published online January 22, 2021
We assessed statistical significance at the 0.05 level. Stata 16 (College Station, TX) was used to analyze the data.

\section{RESULTS}

Between February 1 and October 10, 2020, the NCHS reported 203,043 COVID-19 deaths in the USA: 8312 Asians, 363 NHPIs, 104,846 Whites, 41,663 Blacks, 43,107 Latinx, 2039 AIANs, and 574 multi-racial individuals. Across all ages and race/ethnicity groups, the attributable mortality from COVID19 overall was $9.2 \%$. The rates among Asians, Latinx, Blacks, AIANs, and NHPIs were significantly higher than that of nonHispanic Whites (6.5\%) (Fig. 1).

The patterns of the association between COVID-19 attributable mortality and age differ across racial/ethnic groups (Fig. 2). Asians had statistically higher attributable mortality compared to non-Hispanic Whites between ages 45 and older. NHPIs had the highest attributable mortality between ages 15 and 24, and had higher rates than non-Hispanic Whites between ages 45 and 84 . AIANs had higher rates than nonHispanic Whites between ages 45 and 84. Blacks had higher rates than non-Hispanic Whites for those ages 35 and older. Latinx had higher attributable mortality rates than nonHispanic Whites from age 15 across all ages.

\section{DISCUSSION}

Our findings are consistent with others reporting the disproportionate burden of COVID-19 among Latinx and Black Americans, but our analysis raises concern about the lack of policy attention of the COVID-19 burden among Asians and NHPIs. Asians, Latinx, and Black Americans had the three highest COVID-19 attributable deaths, with frequencies at least twice that of Whites. Similar to many communities of color, Asians and NHPIs may have worse COVID-19 mortality because they disproportionately work in healthcare and other essential jobs, have underlying medical comorbidities, and are more likely to live in denser, multi-generational households. ${ }^{2,5}$ The higher attributable death among Asians may also be due to a heightened xenophobia that may have discouraged Asians from seeking timely testing and treatment, particularly among $60 \%$ of the population who are immigrants and $23 \%$ who are limited English proficient. ${ }^{6}$

Our analysis uncovering Asian and NHPI attributable COVID-19 deaths by age is significant as this disaggregated 


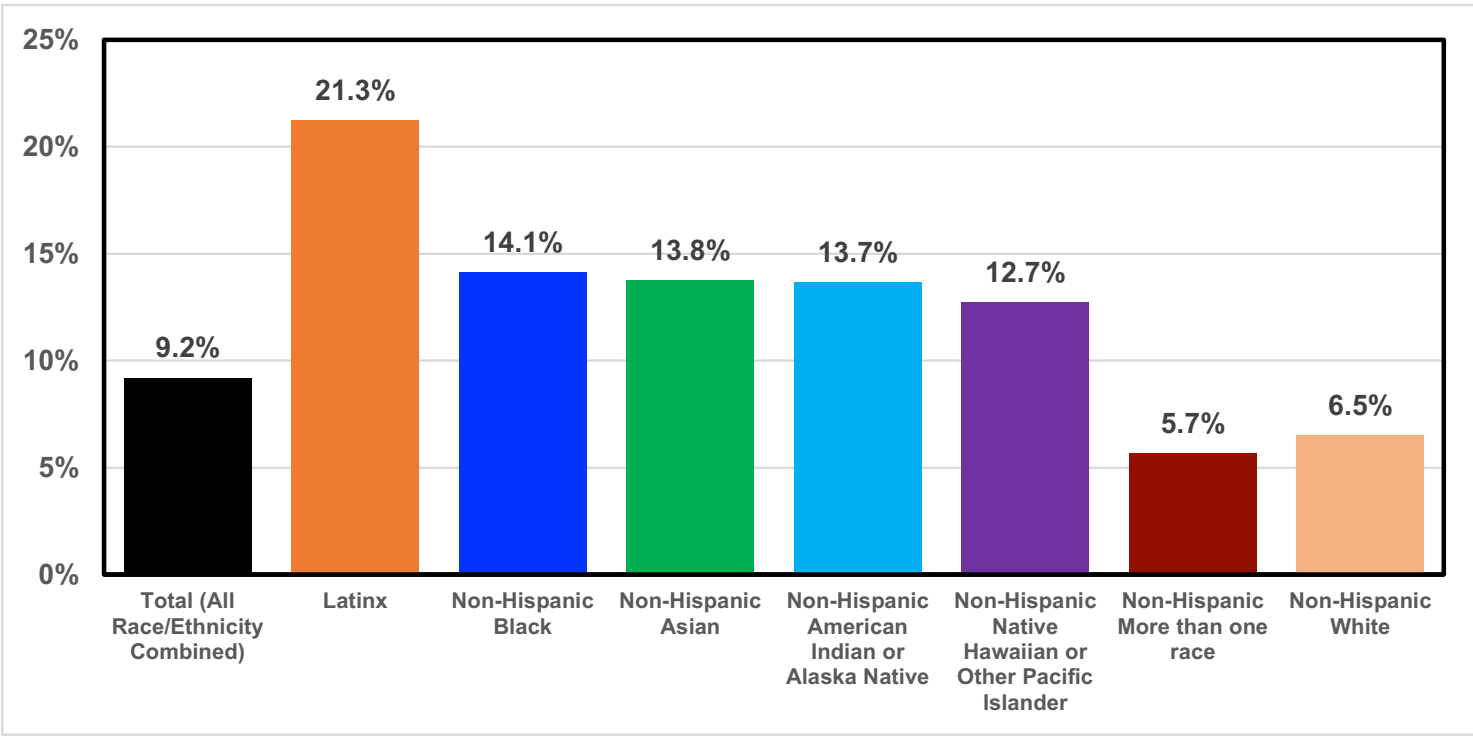

Figure 1 Attributable mortality* of COVID-19 in the USA by race/ethnicity groups among all ages reported to the National Center of Health Statistics between February 1 and October 10, 2020. *Attributable mortality = proportion of COVID-19 deaths out of the total number of deaths from all causes including COVID-19 for each group of focus. Total (includes all race/ethnicity combined, with those with "Unknown" race/ethnicity group). COVID-19 death data from NCHS come from death certificate data and may differ from other published resources.

data is not available in many public datasets. The Office of Management and Budget requires that agencies receiving federal funding report Asians as a separate category from
NHPIs, while some states continue to categorize Asians and NHPIs as one category, and others aggregate Asians, NHPIs, and AIANs under "Other." We must correct this. Over the

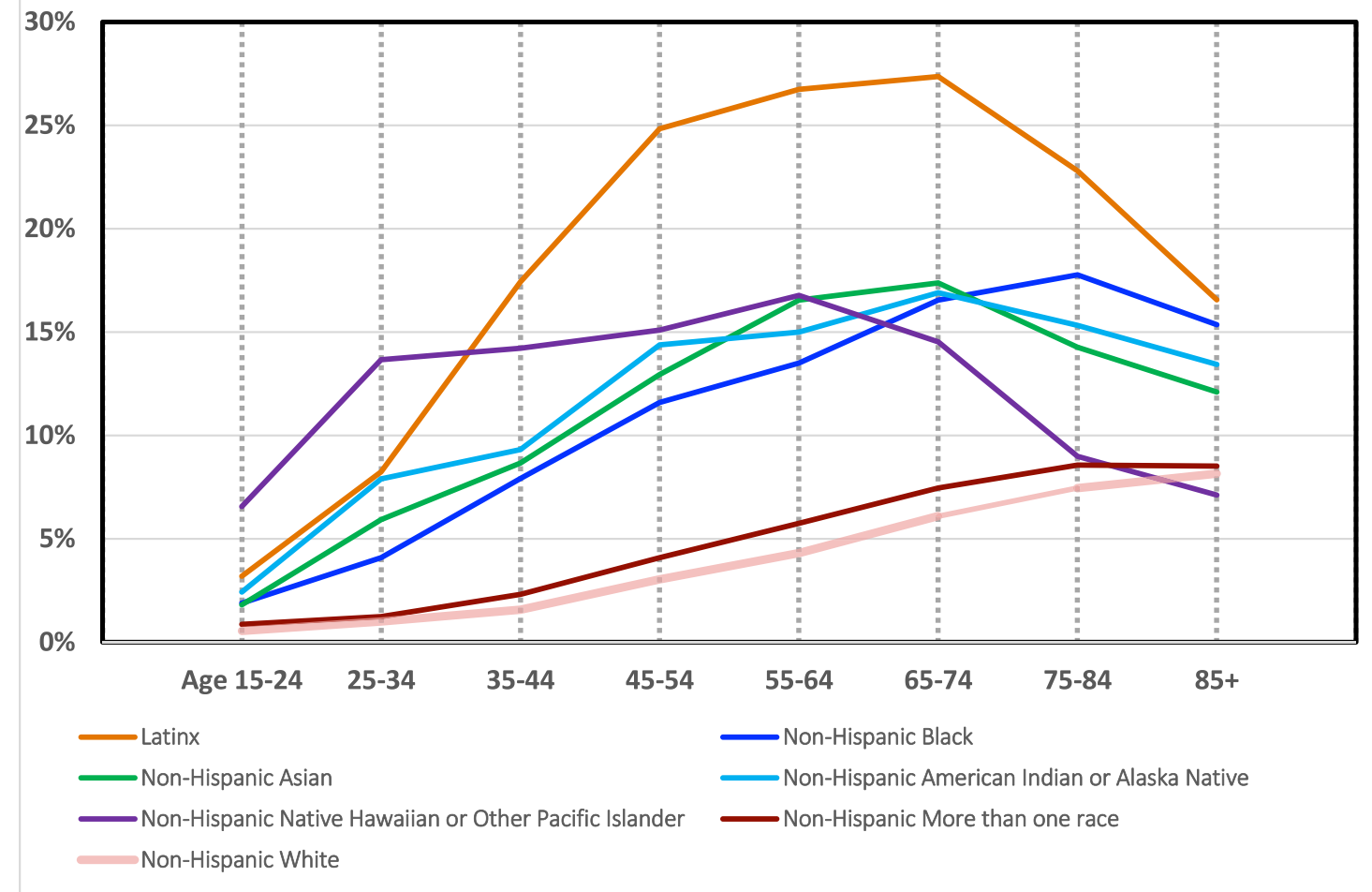

Figure 2 Attributable mortality of COVID-19 in the USA across age groups by race/ethnicity groups among individuals 15 years and older reported to the National Center of Health Statistics between February 1 and October 10, 2020. When examining the attributable mortality by specific age groups by race/ethnicity, the coefficients of variation were greater than $30 \%$ in the age groups under 15 years of age, likely because of the relatively small number of COVID-19 deaths in these age groups. Therefore, we limited our results of attributable mortality across racial/ ethnic groups for each age group to those 15 years of age and older. COVID-19 death data from NCHS come from death certificate data and may differ from other published resources. 
course of this pandemic, public health departments must provide access to COVID-19 testing, case, death, hospitalization data by primary language, and disaggregated race/ethnicity. This would improve prevention, mitigate spread, and provide better healthcare for Asians and NHPIs whose lives and livelihoods have been overlooked and harmed by incomplete or missing data.

Janet N. Chu, $M D, M P H^{1,2}$

Janice $Y$. Tsoh, $\mathrm{Ph} \mathrm{D}^{2,3}$

Elena Ong, PHN, MS ${ }^{4}$

Ninez A. Ponce, $P h D, M P P^{5}$

${ }^{1}$ Department of Medicine, University of California San Francisco School of Medicine,

San Francisco, CA, USA

${ }^{2}$ Asian American Research Center on Health,

San Francisco, CA, USA

${ }^{3}$ Department of Psychiatry and Behavioral Sciences, University of California San Francisco School of Medicine,

San Francisco, CA, USA

${ }^{4}$ Ong \& Associates,

Los Angeles, CA, USA

${ }^{5}$ UCLA Fielding School of Public Health/UCLA Center for Health Policy Research, University of California Los Angeles,

Los Angeles, CA, USA

Corresponding Author: Janet N. Chu, MD, MPH; Department of Medicine, University of California San Francisco School of Medicine, San Francisco, CA, USA (e-mail: janet.chu@ucsf.edu).

Authors' Contributions NP and EO made substantial contributions to the concept and design. JT, JC, and NP made substantial contributions to the data analysis and interpretation. JC led the writing; JT, EO, and NP revised it critically.
Funding J. Chu is funded in part by a National Research Service Award fellowship training grant (T32HP19025). N. Ponce is funded by the Robert Wood Johnson Foundation.

\section{Compliance with Ethical Standards:}

Conflict of Interest: The authors declare that they do not have a conflict of interest.

Ethics Statement: The corresponding author has had full access to all aspects of the research and writing process and takes final responsibility for the paper. All authors listed have contributed sufficiently to the project to be included as authors, and all those who are qualified to be authors are listed in the author byline.

\section{REFERENCES}

1. COVID-19 Map. Johns Hopkins Coronavirus Resource Center. Accessed July 4, 2020. https://coronavirus.jhu.edu/map.html

2. Webb Hooper M, Nápoles AM, Pérez-Stable EJ. COVID-19 and Racial/ Ethnic Disparities. JAMA. Published online May 11, 2020. https://doi. org/10.1001/jama.2020.8598

3. Calgary O. Provisional Death Counts for Coronavirus Disease (COVID-19): Weekly State-Specific Data Updates | Data | Centers for Disease Control and Prevention. Accessed August 4, 2020. https://data.cdc.gov/NCHS/ Provisional-Death-Counts-for-Coronavirus-Disease-C/pj7m-y5uh

4. von Cube M, Timsit J-F, Schumacher M, Motschall E, Schumacher M. Quantification and interpretation of attributable mortality in core clinical infectious disease journals. Lancet Infect Dis. Published online September 8, 2020. https://doi.org/10.1016/S1473-3099(20)30485-0

5. Count Native Hawaiian And Pacific Islanders In COVID-19 Data-It's An OMB Mandate | Health Affairs. Accessed October 20, 2020. https://www. healthaffairs.org/do/10.1377/hblog20200825.671245/full/

6. Yan B, Ng F, Chu J, Tsoh J, Nguyen T. Asian Americans Facing High COVID-19 Case Fatality. Health Affairs Blog

Publisher's Note: Springer Nature remains neutral with regard to jurisdictional claims in published maps and institutional affiliations. 